\title{
Patrones estacionales de abundancia en el Golfo Nuevo y migración a lo largo de la plataforma Argentina de cazones (Galeorhinus galeus [Linnaeus 1758]) y gatopardos (Notorynchus cepedianus [Péron 1807]) (Argentina)
}

\author{
Alejo Irigoyen $^{1, \otimes}$; Carolina Sibbald ${ }^{2}$; Mariano Cuestas ${ }^{3}$; Franco CRistiani ${ }^{2}$ \\ \& GASTÓN TROBBIANI ${ }^{1}$
}

1. Centro Nacional Patagónico-Consejo Nacional de Investigaciones, Científicas y Técnicas (CENPAT-CONICET). Puerto Madryn. 2. Universidad Nacional de la Patagonia San Juan Bosco. Puerto Madryn. 3. Zep Ltd. Puerto Madryn, Chubut, Argentina.

\begin{abstract}
Resumen. Conocer los patrones de movimiento de una especie tiene implicancias sustanciales para su manejo y conservación, en particular en aquellas que realizan migraciones de gran escala. El objetivo de este trabajo fue estudiar los patrones estacionales de abundancia de cazones y gatopardos en el Golfo Nuevo, provincia de Chubut, y sus patrones migratorios a lo largo de la plataforma argentina, por medio de un plan de marcado y recaptura convencional. Se capturaron y marcaron un total de 13 gatopardos y 228 cazones a lo largo de un ciclo anual completo. A partir del análisis de datos de captura por unidad de esfuerzo (CPUE) se encontró que el cazón presentó un patrón estacional marcado, con una abundancia mayor desde mediados del verano y durante el otoño; la abundancia fue mínima en primavera, cuando se registraron dos recapturas de cazones al norte del sitio de marcado $\left(38^{\circ}\right.$ y $\left.40^{\circ} \mathrm{S}\right)$. Estos resultados constituyen la primera evidencia directa que apoya la hipótesis de que existe una población única de cazones en el Atlántico Sur. Sin embargo, la detección de cazones machos en el sitio de estudio durante todo el año indica que al menos una parte de la población podría pasar los meses fríos del año en el sur del área de distribución. En el caso del gatopardo, si bien fue muy bajo el número de ejemplares capturados, la ausencia de la especie durante los meses fríos del año indica que podría tener una estacionalidad similar a la del cazón.
\end{abstract}

[Palabras clave: movimiento animal, marcado convencional, recaptura, conservación]

\begin{abstract}
Aвstract. Seasonal patterns in Golfo Nuevo and migration on the Argentine shelf of school (Galeorhinus galeus [Linnaeus 1758]) and sevengill (Notorynchus cepedianus [Péron 1807]) sharks (Argentina): Knowing the movement patterns of a certain species has substantial implications for its management and conservation, particularly in species that migrate hundreds to thousands of kilometers each year (e.g., many chondrichtyans). The objective of this paper was to study the seasonal abundance in the Golfo Nuevo and the migratory patterns of the school and sevengill shark across the Argentine shelf through a conventional capture-recapture plan. A total of 13 sevengill and 228 school sharks were tagged during a full annual cycle. From analysis of catch per unit effort we found that the species studied show a strong seasonal pattern in the Golfo Nuevo, with higher abundance from mid-Summer to Autumn and a lower one during Spring, when two school shark recaptures were registered north from the tagging site $\left(38^{\circ}\right.$ and $\left.40^{\circ} \mathrm{S}\right)$. These results represent the first direct evidence supporting the hypothesis of a unique school shark population in the South Atlantic Ocean. Nevertheless, the detection of male school sharks in the study site all year round indicates that at least part of the population could be spending the cold months of the year south of its distribution area. In the case of the sevengill shark, despite that few individuals were captured, the absence of the species during cold months suggest a seasonality similar to the school shark.
\end{abstract}

[Keywords: animal movement, conventional tagging, recapture, conservation]

\section{INTRODUCCIÓN}

La mayoría de los procesos característicos de la vida de los vertebrados están ligados al movimiento, con causas que operan a múltiples escalas espaciales y temporales (Begon et al. 2006). El modo en que los individuos se desplazan determina sus opciones de supervivencia y reproducción, la distribución y la escala de sus interacciones

Editora asociada: María Semmartin con el medio y, en consecuencia, la estructura y la dinámica de poblaciones, comunidades y ecosistemas (Robledo-Arnuncio \& González Martínez 2009). En los peces, y en especial dentro del grupo de los condrictios, son muchas las especies que realizan migraciones del orden de cientos a miles de kilómetros cada año (Helfman et al. 2009). Conocer sobre los movimientos a gran escala, que en muchos casos atraviesa fronteras políticas, tiene

Recibido: 30 de octubre de 2014, Fin de arbitraje: 16 de enero de 2015, Última versión revisada: 21 de enero, Aceptado: 30 de marzo. 
implicancias de carácter sustancial en lo que a conservación se refiere, ya que las amenazas, las regulaciones y su aplicación varían entre regiones y naciones (Speed et al. 2010).

A escala global, la sobrepesca y la degradación de hábitats afectan marcadamente a los condrictios (Stevens et al. 2000; Ferreti et al. 2010). La Unión Internacional para la Conservación de la Naturaleza (IUCN), a través del Grupo de Especialistas en Tiburones, informó que un cuarto de las especies de tiburones en el planeta se encuentra en peligro de extinción (Dulvy et al. 2014). En Argentina, en el marco del Plan Nacional Para la Conservación y Manejo de Condrictios, si bien se cuenta con información pesquera de algunas especies medianas y chicas de interés comercial (e.g., el gatuzo [Mustelus schimitti] y el pez gallo [Callorhynchus callorhynchus]), la falta de información biológica, ecológica y de capturas es una de las grandes necesidades para evaluar el estado de conservación y generar pautas de manejo de condrictios en general (Wohler et al. 2011).

Las especies estudiadas en este trabajo, el cazón (Galeorhinus galeus [Linnaeus 1758]) y el gatopardo (Notorynchus cepedianus [Péron 1807]), presentan una distribución global en aguas templadas, a excepción del Atlántico Norte y Mediterráneo en el caso del gatopardo. En el Atlántico Sudoccidental se distribuyen desde el sur de Brasil hasta el sur de Argentina (Sadowsky 1970; Guzmán \& Campodónico 1976; Vooren 1997; Góngora 2011). Son muy vulnerables al impacto humano por su longevidad elevada y su baja fecundidad (Lucifora 2003). Si bien para la región no existen estudios específicos sobre patrones migratorios o movimiento de estas especies, en el caso del cazón, a partir de la complementariedad de datos pesqueros y de análisis del desarrollo embrionario entre sitios del sur de Brasil y la costa Argentina, se cree que existiría una población única para todo el Atlántico sur (Lucifora et al. 2004). Esta población pasaría los meses fríos del año al norte del área de distribución y migraría hacia el sur durante la primavera y verano. Además, se cree que los machos permanecerían en zonas costeras durante las épocas frías, mientras que las hembras habitarían zonas profundas alejadas de la costa (Peres \& Vooren 1991; Vooren 1997). El cazón fue categorizado en estado crítico por la IUCN para la región del Atlántico sur. Dicha clasificación se basó en una disminución de $80 \%$ de la captura por unidad de esfuerzo en pesquerías de arrastre en la década del noventa. Por otro lado, el gatopardo está categorizado como deficiente de datos (Walker et al. 2006), ya que no se cuenta con información pesquera o investigaciones que permitan hacer inferencias sobre su estado de conservación (Compagno 2005). Sin embargo, la abundancia actual de estas especies es percibida por pescadores deportivos y artesanales (mayores a 40 años de edad) en $\sim 20 \%$ de la abundancia que existía al comienzo de sus carreras (Irigoyen \& Trobbiani, en prensa).

El objetivo de este trabajo fue describir los patrones de abundancia estacionales en el golfo Nuevo y migratorios de cazones y gatopardos a lo largo de la plataforma argentina, por medio de un plan de marcado y recaptura convencional. Se utilizaron marcas externas que permitieron que la información sobre el movimiento sea recabada cuando un pescador informa el lugar y la fecha de la captura. Además, se estableció como objetivo complementario el poner a punto un método de captura y marcado efectivo y de bajo costo.

\section{MATERIALES Y MÉTODOS}

La captura y marcado de cazones y gatopardos se realizó en el sector sudoeste del Golfo Nuevo $\left(\sim 42.5^{\circ} \mathrm{S}\right)$, entre 80 y $110 \mathrm{~m}$ de profundidad (Figura 1). Este sitio forma parte del área de Península Valdés, declarada recientemente Patrimonio Mundial de la Humanidad por las Naciones Unidas en función de su relevancia desde el punto de vista de la conservación, y en particular por la gran concentración de aves y mamíferos marinos.

Los muestreos consistieron en salidas de pesca diarias. Se llevaron a cabo durante un ciclo anual completo, desde enero de 2011 hasta enero de 2012 (un total de 17 salidas en el período). Si bien inicialmente se planeó un muestreo regular a lo largo del año con una periodicidad mínima mensual, durante los meses de mayor número de capturas se realizaron salidas de pesca adicionales a fin de aumentar el número total de individuos marcados. Por otro lado, el menor número de salidas realizadas durante el invierno y primavera se debió a condiciones climáticas adversas.

Se utilizó un espinel de entre 500 y 1200 m de largo, que consistió en anzuelos biodegradables ("Goldfish-Octopus Hook" de tipo 10/0 serie 92553) colocados sobre una línea madre (7 mm de diámetro) cada $10 \mathrm{~m}$ de distancia, mediante monofilamento de $0.8 \mathrm{~mm}$ de diámetro y $1 \mathrm{~m}$ de largo. Como carnada se utilizó magrú (Scomber japonicus) los primeros dos meses, savorín (Seriorella porosa) en una ocasión 


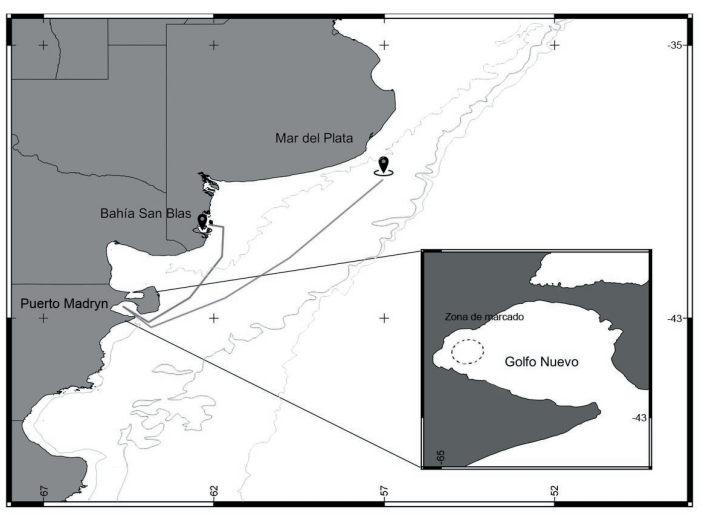

Figura 1. Atlántico Sur, los símbolos en forma de círculos indican los lugares de recaptura de cazones (Galeorhinus galeus) en el Canal Culebra $\left(40.2^{\circ} \mathrm{S}\right.$ y $62.1^{\circ} \mathrm{O}-15 \mathrm{~m}$ de profundidad), Bahía San Blas, y frente a la costa de Mar del Plata $\left(38.4^{\circ} \mathrm{S}\right.$ y $57.3^{\circ} \mathrm{O}-40 \mathrm{~m}$ de profundidad). Se indican las isobatas de 50, 100 y $200 \mathrm{~m}$ de profundidad (de izquierda a derecha). En el recuadro se detalla el Golfo Nuevo y zona de pesca ( 100 m de profundidad).

Figure 1. South West Atlantic Ocean, the symbols indicate the recapture locations of school sharks (Galeorhinus galeus) in the Canal Culebra (40.2 ${ }^{\circ} \mathrm{S}$ y $62.1^{\circ} \mathrm{O}-15 \mathrm{~m}$ depth), Bahía San Blas, and offshore of Mar del Plata $\left(38.4^{\circ} \mathrm{S}\right.$ y $57.3^{\circ} \mathrm{O}$ - $40 \mathrm{~m}$ depth). Isobaths of 50, 100 and $200 \mathrm{~m}$ of depth are indicated from left to right. In the panel, a detail of the study site and tagging area ( 100 m depth).

y pejerrey (Odontesthes bonariensis) durante el resto del estudio por razones de mayor disponibilidad y menor costo.

Cada salida de pesca comenzaba al amanecer (entre las 5 y 8:30 am según la época del año), con una tripulación de entre dos y cuatro personas, utilizando una embarcación de tipo semirígido. Al momento de llegar al sitio de pesca (que era establecido al encontrar los $100 \mathrm{~m}$ de profundidad a la menor distancia posible de la costa), el palangre era extendido de forma lineal sobre el fondo, sujetado en los extremos por dos anclas de tipo danford de $2 \mathrm{~kg}$. A diferencia de los espineles comerciales (que separan la línea de anzuelos del fondo mediante pequeños flotadores), en este trabajo el espinel fue tendido directamente sobre el fondo. El motivo de esta modificación del arte de pesca fue disminuir el peso del equipo transportado en las embarcaciones. El número de anzuelos utilizado, y por ende el largo de espinel, dependió en cada caso de la capacidad de carga de la embarcación y de las condiciones climáticas.

El tiempo de espera (tiempo de permanencia de los anzuelos en al agua) fue de dos horas como máximo, de forma precautoria, a partir de pruebas pilotos anteriores al inicio del estudio. Durante estas salidas, el tiempo de espera fue incrementado poco a poco desde $0.5 \mathrm{~h}$ hasta $2 \mathrm{~h}$, y en ningún caso se registró mortalidad, ni daños, ni signos de agotamiento severos en 21 capturas. Al recoger el palangre, los tiburones fueron llevados de forma manual a la embarcación, sujetados del rostro y la cola, o bien sujetados de la zona branquial en ambos lados. En primera instancia se les retiraba el anzuelo de la boca; ante cualquier dificultad, el anzuelo era cortado y retirado por completo. Luego se les colocaba una marca del tipo "dart tags- Ft1-94/floytag" en el sector anterior de la primera aleta dorsal utilizando un punzón pequeño. Se registró el sexo según la presencia o no de claspers (aparato reproductor masculino ubicado en las aletas pélvicas) y se estimó visualmente la talla de cada individuo. El protocolo de manipulación y marcado fue establecido con el objetivo de devolver los tiburones al agua en el menor tiempo posible, en detrimento de obtener medidas precisas de largo, peso o realizar toma de muestras biológicas. Las marcas utilizadas contaban con un número único, una leyenda con el contacto del investigador a cargo del proyecto (teléfono celular y correo electrónico) y el ofrecimiento de una recompensa por reportar la captura del ejemplar marcado.

Se estimó la abundancia relativa de cazones y gatopardos a partir del cálculo de CPUE a partir del número de individuos capturados por el número de anzuelos empleados y por tiempo de espera en horas (individuos/anzuelos/horas).

\section{Resultados}

Se capturaron y marcaron un total de 13 gatopardos (7 hembras, 3 machos y 3 no identificados) y 228 cazones (129 hembras, 98 machos y 1 no identificado). Estas especies representaron el $84 \%$ de la captura total. El resto de condrictios capturados $(n=30)$ representó el $10 \%$ de la captura total y fueron devueltos al mar en buen estado. El restante $6 \%$ de la captura estuvo compuesto por merluza (Merluccius hubbsi) y peces de arrecife (Pseudopercis semifasciata, Acanthistius patachonicus, Salilota australis y Sebastes oculatus). Los mayores valores de CPUE para ambas especies se registraron durante el verano y el otoño, y los mínimos en invierno y primavera (Figura 2). En el caso del gatopardo, los valores de CPUE fueron bajos, y con captura nula en invierno y primavera. La proporción de sexos de cazones capturados varió durante las estaciones del año: en verano y otoño predominaron las hembras, mientras que en invierno y primavera, los machos (Figura 3).

Se registró la recaptura de dos cazones, informadas por vía telefónica. Ambos individuos recapturados habían sido marcados en marzo de 2011. La primer recaptura, en noviembre del mismo año de marcado, fue realizada a $487 \mathrm{~km}$ en línea recta de la zona de marcado por un guía de pesca deportiva en el Canal Culebra, Bahía San Blas $\left(40.2^{\circ} \mathrm{S}\right.$ y $\left.62.1^{\circ} \mathrm{O}\right)$ a $15 \mathrm{~m}$ de profundidad. La segunda 

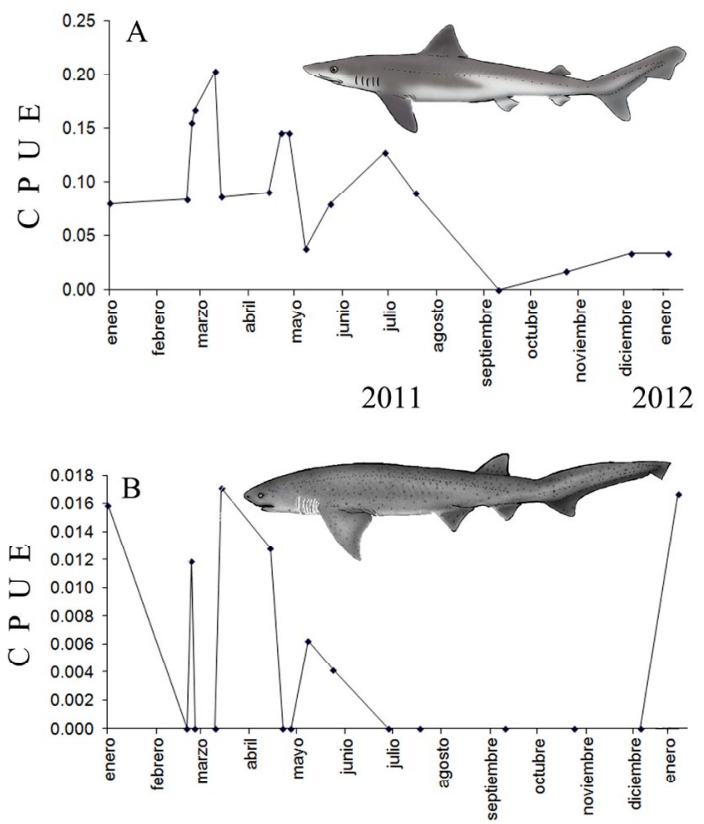

2011

2012

Figura 2. Captura Por Unidad de Esfuerzo (CPUE) calculado por salida de pesca (Nro. de capturas x Nro. anzuelos $\left.^{-1} \mathrm{x} \mathrm{h}^{-1}\right)$. A) cazón (Galeorhinus galeus) y B) gatopardo (Notorynchus cepedianus).

Figure 2. Catch per unit effort (CPUE) calculated for each fishing trip (Nr. captures $\times$ Nr. hooks ${ }^{-1} \mathrm{x} \mathrm{h}^{-1}$ ). A) School shark (Galeorhinus galeus) and B) Sevengill shark (Notorynchus cepedianus).

recaptura, en diciembre del mismo año de marcado, fue realizada a $847 \mathrm{~km}$ en línea recta de la zona de marcado por un pesquero de arrastre frente a la costa de Mar del Plata (38.4 ${ }^{\circ}$ S y $57.3^{\circ} \mathrm{O}$ ) a $40 \mathrm{~m}$ de profundidad (Figura 1 ). En diciembre de 2012, el número de teléfono grabado en las marcas quedó inhabilitado por razones ajenas al control de los investigadores

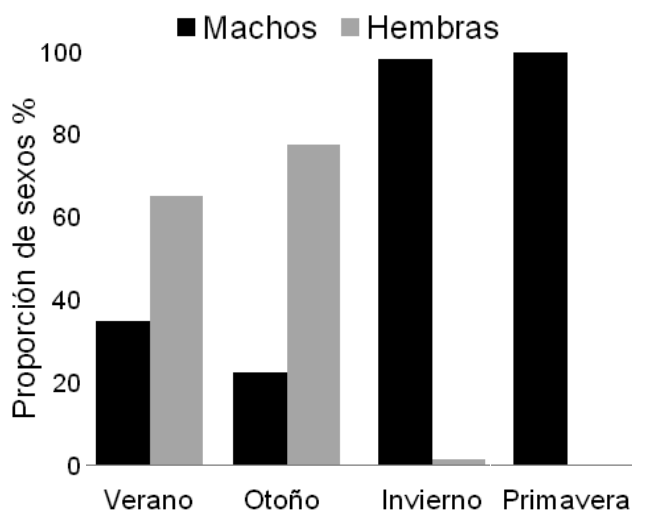

Figura 3. Proporción de sexos (\%) de cazones capturados en las distintas estaciones del año.

Figure 3. Sex ratio (\%) of school sharks captured during the different seasons of the year. y no resultó posible redireccionar llamadas a un nuevo contacto.

El bajo número de salidas en las que se utilizó como carnada magrú y savorín no permitió detectar diferencias en la capturabilidad con respecto a la carnada más utilizada que fue el pejerrey. Sin embargo, durante la salida de pesca en la cual se utilizó savorín se obtuvo un valor de CPUE bajo (en relación a las otras salidas de dicha época del año), posiblemente por lo inadecuado de la misma, ya que se desprendía fácilmente del anzuelo.

\section{DisCUSIÓN}

El cazón (Galeorhinus galeus) mostró un fuerte patrón estacional en el Golfo Nuevo, con una mayor abundancia desde mediados del verano y durante el otoño, y mínima en primavera, época del año en la que se registraron las dos recapturas de cazones al norte del sitio de marcado. A partir de mediados de invierno y durante la primavera sólo se detectó la presencia de cazones machos en baja abundancia. En el caso del gatopardo, si bien fue muy bajo el número de ejemplares capturados y no se realizaron recapturas, su ausencia durante los meses fríos indica que podría tener una estacionalidad similar a la del cazón en el Golfo Nuevo. A mayor escala, para el Atlántico Sur, pescadores deportivos y artesanales de distintas regiones del país reportaron consistentemente una estacionalidad marcada en las capturas, en coincidencia con las del cazón, que durante los meses fríos del año se concentran en la costa norte del país y durante el verano y comienzo del otoño, en el sur (Irigoyen \& Trobbiani, en prensa). Esto implicaría que al menos los adultos de gatopardo realizan grandes migraciones por la plataforma argentina a lo largo del año, como ya fue reportado para la especie en otras regiones del mundo (Barnett et al. 2011).

Los resultados para el cazón concuerdan con los datos previos para la zona de estudio (Elías et al. 2005). A partir del análisis biológico de capturas y datos de CPUE provenientes de pesca artesanal y experimental, Elías (2005) concluyó que la especie arriba a la Patagonia norte durante el verano y que los cardúmenes están formados principalmente por machos maduros y hembras inmaduras y maduras en el primer y segundo año de no gravidez con el objetivo principal de alimentarse. Por otro lado, la autora documentó que las hembras se encuentran a mayor profundidad que los machos, con un máximo de segregación 
por sexos en las profundidades en las que se realizó este trabajo. El patrón estacional en el golfo Nuevo y las dos recapturas obtenidas de cazones en este trabajo apoyan la hipótesis de que existiría una población única de la especie para todo el Atlántico sur, la cual migraría entre el sur de Brasil y el de Argentina a la largo del ciclo anual. Vale remarcar que las dos recapturas representan la primera evidencia directa de dicha hipótesis. Por otro lado, la captura de cazones machos durante todo el año en el Golfo Nuevo indicaría que, en parte, la población de cazones podría tener una estrategia alternativa y pasar una mayor parte del ciclo migratorio en el sur del área de distribución. Esta presencia de machos en aéreas costeras durante la primavera se ajusta a una descripción más general sobre el patrón migratorio de la especie (aunque realizada para la región norte del área de distribución de la especie): las hembras pasarían los meses fríos del año en zonas profundas alejadas de la costa, y los machos, en zonas costeras (Peres \& Vooren 1991; Vooren 1997).

El porcentaje de recapturas de este trabajo fue bajo $(0.87 \%)$ en comparación con otros programas de recaptura con marcas convencionales (Kohler \& Turner 2001; Pérez et al. 2014). A pesar de que, en general, las recapturas en este tipo de planes de marcado son bajas (Kohler \& Turner 2001), la inhabilitación del número de teléfono impreso (a los pocos días luego de recibir el segundo reporte de recaptura) probablemente haya representado un sesgo significativo, a pesar de que siguió existiendo un correo electrónico válido.

Finalmente, la relación satisfactoria de esfuerzo y costo de este trabajo en relación al número de tiburones marcados alienta a futuros esfuerzos y a establecer planes de marcado de largo plazo a fin de recabar mayor información sobre las rutas migratorias de las especies de estudio y el monitoreo de abundancias relativas mediante análisis de CPUE.

Agradecimientos: Deseamos agradecer a los pescadores artesanales J. Ascorti y L. de Francesco por el asesoramiento en relación a las tareas de pesca. A M. Lupiano, G. Bravo, J. Lacelloti, L. Pozzi y C. Arwuch por su ayuda en las tareas de campo. El trabajo fue financiado por PADI-Foundation.

\section{BIBLIOGRAFÍA}

Barnet, A; KG Abrantes; JD Stevens \& JM SEMmens. 2011. Site fidelity and sex-specific migration in a mobile apex predator: implications for conservation and ecosystem dynamics. Anim. Behav., 81:1039-1048.
BEgOn, M; CR TOWNSEND \& JL HARPER. 2006. Ecology 4th ed. Blackwell Publishing, Victoria, Australia.

Compagno, LJV. 2005. Notorynchus cepedianus. IUCN 2013. IUCN Red List of Threatened Species. Version 2013.1.

Dulvy, NK; SL Fowler; JA Musick; RD Cavanagh; PM KYNE; ET AL. 2014. Extinction risk and conservation of the world's sharks and rays. eLife, 3:e00590.

Elías, I; A Rodríguez; E Hasan; MV Reina \& R Amoroso. 2005. Biological Observations of the Tope Shark, Galeorhinus galeus, in the Northern Patagonian Gulfs of Argentina. J. Northw. Atl. Fish. Sci., 35:261-265.

Ferretti, F; B Worm, GL Britten; MR Heithaus \& HK Lotze. 2010. Patterns and ecosystem consequences of shark declines in the ocean. Ecol. Lett., 13:1055-1071.

GóngOra, ME; ND BOvCON \& PDCOCHIA. 2009. Fish bycatch in the Patagonian shrimp fishery Pleoticus muelleri Bate 1888. Rev. Biol. Mar. Oceanogr., 44:583-593.

GuzMán, L \& I CAmpodónICo. 1976. Notorynchus cepedianus (Péron, 1807) en la entrada oriental del Estrecho de Magallanes (Elasmobranchii, Hexanchiidae). Anales del Instituto de la Patagonia, 7:207-210.

Helfman, GS; BB Collette; DE Facey \& BW Bowe. 2009. The Diversity of Fishes. Biology, Evolution, and Ecology. Wiley-Blackwell, Oxford, UK.

Irigoyen, AJ \& G Trobbiani. 2015. Depletion of trophy large-sized sharks populations of the Argentinean coast, south-western Atlantic: insights from fishers' knowledge. Neotrop. Ichtiol. (En prensa)

KoHLER, NE \& PA TURNER. 2001. Shark tagging: a review of conventional methods and studies. Environ. Biol. Fishes., 60:191-223.

Lucifora, LO; RC Menni \& AH Escalante. 2004. Reproductive biology of the school shark, Galeorhinus galeus, off Argentina: support for a single south western Atlantic population with synchronized migratory movements. Environ. Biol. Fishes, 71:199-209.

LuCIFORA, LO. 2003. Ecología y Conservación de Los Grandes Tiburones Costeros de Bahía Anegada, Provincia de Buenos Aires, Argentina. Tesis Doctoral, Universidad Nacional de Mar del Plata, Mar del Plata, Argentina.

Peres, MB \& CM Vooren. 1991. Sexual development, reproductive cycle, and fecundity of the school shark Galeorhinus galeus off southern Brazil. Fish. Bull., 89:655-667.

Pérez, M; M Braccini \& MB Cousseau. 2014. Uso de marcas convencionales en un país con escasa experiencia en marcación de peces marinos. Lat. Am. J. Aquat. Res., 42(1):258-263.

Robledo-Arnuncio, JJ \& SC GonzÁlez Martínez. 2009. Marcadores moleculares y ecología del movimiento. Ecosistemas, 18(1):44-51.

SADOWSKY, V. 1970. First record of broad-snouted sevengilled shark from the Cananéia, Brazil. Boletim do Instituto Paulista de Oceanografia, 18:33-35.

Speed, CW; IC FIELd; MG MeEkan \& CJA Bradshaw. 2010. Complexities of coastal shark movements and their implications for management. Mar. Ecol. Prog. Ser., 408:275-293.

Stevens, JD; R Bonfil; NK Dulvy \& P WAlker. 2000. The effects of fishing on sharks, rays and chimaeras (Chondrichthyans), and the implications for marine ecosystems. ICES J. Mar. Sci., 57:476-494.

Vooren, CM. 1997. Demersal elasmobranchs. Pp. 141146 en: Seeliger, U; C. Odebrecht \& JP Castello (eds.). Subtropical Convergence Environments: The Coast and Sea in the Southwestern Atlantic. Springer-Verlag, Heidelberg, Alemania.

Walker, TI; RD Cavanagh; JD Stevens; AB Carlisle; G Chiramonte; A Domingo A; et al. 2006. Galeorhinus galeus. IUCN 2011. IUCN Red List of Threatened Species. Version 2011.1. 